\title{
Fibroblasts facilitate re-epithelialization in wounded human skin equivalents
}

\author{
Abdoelwaheb El Ghalbzouri ${ }^{1,2}$, Paul Hensbergen ${ }^{1}$, Sue Gibbs ${ }^{1,4}$, Johanna Kempenaar ${ }^{1}$, \\ Roel van der Schors ${ }^{3}$ and Maria Ponec ${ }^{1}$ \\ ${ }^{1}$ Department of Dermatology, Leiden University Medical Center, Leiden, The Netherlands; ${ }^{2}$ Isotis.N.V. \\ Bilthoven, The Netherlands and ${ }^{3}$ Department of Molecular Neurobiology, Free University, Amsterdam, \\ The Netherlands
}

\begin{abstract}
The re-epithelialization of the wound involves the migration of keratinocytes from the edges of the wound. During this process, keratinocyte migration and proliferation will depend on the interaction of keratinocytes with dermal fibroblasts and the extracellular matrix. The present study aimed to investigate (1) the role of fibroblasts in the re-epithelialization process and on the reconstitution of the dermal-epidermal junction (DEJ) and (2) differential protein expression during re-epithelialization. For both purposes, three-dimensional human skin equivalents (HSE) were used. A full-thickness wound in HSE was introduced by freezing with liquid nitrogen and a superficial wound by linear incision with a scalpel. The closure of the wound in the absence or presence of exogenous growth factors was followed by monitoring the rate of re-epithelialization and regeneration of the DEJ. The results obtained in this study demonstrate that fibroblasts facilitate wound closure, but they differentially affected the deposition of various basement membrane components. The deposition of laminin $\mathbf{5}$ at the DEJ was delayed in superficial wounds as compared to the full-thickness wounds. During freeze injury, some basement membrane (BM) components remain associated with the dermal compartment and probably facilitate the BM reconstitution. The re-epithelialization process in full-thickness but not in superficial wounds was accelerated by the presence of keratinocyte growth factor and especially by epidermal growth factor. In addition, we have examined the deposition of various basement membrane components and the differences in protein expression in a laterally expanding epidermis in uninjured HSE. Laminin 5, type IV and VII collagen deposition was decreased in the laterally expanding epidermis, indicating that the presence of these proteins is not required for keratinocyte migration to occur in vitro. Using twodimensional polyacrylamide gel electrophoresis, we have identified DJ-1, a protein not earlier reported to be differently expressed during the epithelialization process of the skin.

Laboratory Investigation (2004) 84, 102-112, advance online publication, 20 November 2003; doi:10.1038/labinvest.3700014
\end{abstract}

Keywords: re-epithelialization; 2D-PAGE; DJ-1; laminin 5; dermal fibroblasts; keratinocytes

Our skin is the first line of defense against disease and skin damage. The body is capable of closing injuries spontaneously in order to restore the protective covering function of the skin as quickly as possible. ${ }^{1-4}$ Both wound contraction and reepithelialization from the margins of the wound play an important role in wound closure. ${ }^{5-7} \mathrm{Re}-$ epithelialization is achieved by keratinocyte proliferation and migration over an extracellular matrix. ${ }^{4,8}$ In addition, the full regeneration of a functional

Correspondence: Dr M Ponec, Department of Dermatology, Leiden University Medical Center, Sylvius Laboratory, PO Box 9503, 2300 RA Leiden, The Netherlands.

E-mail: m.h.ponec-waelsch@lumc.nl

${ }^{4}$ Current address: Department of Dermatology, Free University, Amsterdam, The Netherlands.

Received 08 July 2003; revised 20 August 2003; accepted 21 August 2003; published online 20 November 2003 epidermis depends on the reconstitution of the dermal-epidermal junction (DEJ), which anchors the epidermis to the dermis. ${ }^{9,10}$ The networks of type IV collagen, laminin, perlecan and nidogen are important elements in serving the mechanical stability of the DEJ. ${ }^{11}$ The basement membrane (BM) has been shown to be an active regulator in epithelial-mesenchymal interactions during epithelial cell development. ${ }^{12}$ Wound closure has been studied both in vivo and in vitro. ${ }^{13-19}$ One of the main questions still to be answered is how the keratinocytes are stimulated to migrate into a specific direction and how the reorganization of the cell network necessary for this motion is induced. Furthermore, proteins that direct these processes are still unknown. Fibroblasts have been found to exert profound effects on keratinocyte proliferation and differentiation and on the deposition of basement proteins. ${ }^{20}$ Only in the presence of 
fibroblasts the normalization of the epidermal differentiation program and deposition of laminin 5 , collagen type IV and VII at the dermal/epidermal junction occurred. We can speculate that also during wound-healing processes fibroblasts play an essential role, as it is well established that in vivo the closure of superficial wounds, in which only the epidermis is injured, is faster than in full-thickness wounds, where both the epidermal and dermal components are injured. As it is very difficult to examine the role of fibroblasts on the wound-closure process and on the deposition of basement membrane proteins in vivo, a wound-healing model is presented. This model consists of a three-dimensional human skin equivalent (HSE) model with epidermal architecture closely mimicking that of the native tissue. ${ }^{21-26}$ The full-thickness or superficial epidermal wounds were induced by freezing with liquid nitrogen or by linear incision with a scalpel, respectively. The closure of the wound in the absence or presence of exogenous growth factors was followed by monitoring the rate of re-epithelialization and deposition of basement membrane proteins. In addition, to follow the changes in protein profile during the re-epithelialization process, the epidermal protein profiles were analyzed by two-dimensional polyacrylamide gel electrophoresis (2D-PAGE).

\section{Material and methods}

\section{Cell Culture}

A culture of normal human keratinocytes (NHK) was established from human breast skin, as described earlier. ${ }^{23}$ The keratinocyte medium used for the culture of NHK consisted of three parts Dulbecco's modified Eagle medium and one part Ham's F12 medium supplemented with $5 \%$ HyClone calf serum (Greiner, Nurtingen, Germany), $1 \mu \mathrm{M}$ hydrocortisone, $1 \mu \mathrm{M}$ isoproterenol, $0.1 \mu \mathrm{M}$ insulin (Sigma-Aldricht, Zwijndrecht, The Netherlands). For experiments, secondary cultures were used.

The culture of human dermal fibroblasts was established as described earlier. ${ }^{23}$ The fibroblasts were cultured in DMEM, supplemented with 5\% FCS (Gibco), penicillin (100 IU/ml) and streptomycin $(100 \mu \mathrm{g} / \mathrm{ml})$ (ICN Biomedicals, Inc). Passages two to five were used for the experiments.

\section{Dermal Equivalents}

Hydrated collagen gels were prepared, as described by Smola et al, ${ }^{27}$ using $4 \mathrm{mg} / \mathrm{ml}$ collagen solution isolated from rat-tails. Briefly, the collagen solution was mixed at $4^{\circ} \mathrm{C}$ with fibroblast suspension to reach a final density of $1 \times 10^{5}$ cells $/ \mathrm{ml}$. Subsequently, $2.5 \mathrm{ml}$ of collagen suspension was pipetted onto a filter insert (six wells plate, Costar). After 15 min of incubation at $37^{\circ} \mathrm{C}$ in a humidified atmosphere containing $5 \% \mathrm{CO}_{2}$ to allow gel polymerization, $2 \mathrm{ml}$ of culture medium was added and the collagen matrices were subsequently incubated overnight. In some experiments, $20 \mathrm{ml}$ of collagen mixture was pipetted onto a large filter insert (75 mm diameter, Costar).

\section{Reconstruction of Epidermis}

For the generation of a reconstructed epidermis, secondary keratinocyte cultures $(1 \mathrm{ml}$ containing $1 \times 10^{6}$ keratinocytes) were seeded in the central area (using a plastic ring with a diameter $24 \mathrm{~mm}$ ) of collagen matrices $(75 \mathrm{~mm}$ diameter) and incubated overnight in keratinocyte medium. When smaller inserts (diameter $2 \mathrm{~cm}$ ) were used, $1 \times 10^{6}$ keratinocytes were seeded in $40 \mu \mathrm{l}$ onto a central part of the collagen matrix. Subsequently, the cultures were fed with the same keratinocyte medium but containing $1 \%$ serum, $1 \times 10^{-5} \mathrm{M}$ L-carnitine, $1 \times 10^{-2} \mathrm{M}$ L-serine, $1 \mu \mathrm{M}$ DL- $\alpha$-tocopherol-acetate and a lipid supplement containing $25 \mu \mathrm{M}$ palmitic acid, $15 \mu \mathrm{M}$ linoleic acid, $7 \mu \mathrm{M}$ arachidonic acid and $2.4 \times 10^{-5} \mathrm{M}$ bovine serum albumin. ${ }^{23}$ At the time of lifting the cultures at the air-liquid interface, the same medium was used except that the serum was omitted, the concentration of linoleic acid was increased to $30 \mu \mathrm{M}$ and $100 \mu \mathrm{g} / \mathrm{ml}$ ascorbic acid phosphate was added. ${ }^{23}$ The lifting to the A/L interface was conducted after overnight incubation and a serum-free medium was used 1 day after airexposure. The cultures were grown for additional 14 days, respectively, at the A/L interface. The culture medium was renewed three times a week. All supplements were purchased at Sigma-Aldrich (Zwijndrecht, the Netherlands).

\section{Re-epithelialization Experiments}

Two different approaches have been used for wounding of the HSE: (1) full-thickness or (2) superficial incisional wounding. For creating a full-thickness wound, a metal bar $(2.5 \mathrm{~mm})$ was first cooled in liquid nitrogen and subsequently applied for about $10 \mathrm{~s}$ on the reconstructed epidermis. For superficial incisional wounding, a scalpel was used by means of which about $3 \mathrm{~mm}$ broad epidermal incision was made. After wounding the cultures, they were further grown for 3,7 or 14 days at the A/L interface in a serum-free medium in the absence or presence of growth factors $(1 \mathrm{ng} / \mathrm{ml}$ epidermal growth factor (EGF) or $5 \mathrm{ng} / \mathrm{ml}$ keratinocyte growth factor (KGF)).

\section{Immunohistochemistry and Immunofluoresence Microscopy}

Harvested cultures were washed in PBS, fixed in $4 \%$ paraformaldehyde, dehydrated and embedded in paraffin. Sections $(5 \mu \mathrm{m})$ were cut, deparaffinized in 
ethanol and rehydrated in preparation for morphological or immunohistochemical analysis. Immunohistochemical analysis of several proteins was performed using $5 \mu \mathrm{m}$ frozen sections, which after sectioning at $-20^{\circ} \mathrm{C}$, were air-dried overnight and fixed in acetone for $10 \mathrm{~min}$. The primary antibodies used in the present study are listed in Table 1. After incubation with primary antibodies, sections were stained with the avidin-biotin-peroxidase complex system (streptABcomplex/HRP, DAKO), as described by the suppliers with the following minor modifications: a phosphate-buffered saline was used instead of Tris-buffered saline. All sections were counterstained with hematoxylin.

\section{Assessment of Fibroblast Viability}

After wounding, the tissue was first incubated for $2 \mathrm{~min}$ in $5 \%$ trypan blue (Merck, Darmstadt, Germany) in PBS and thereafter washed with PBS. Photographs of the dermal matrix were taken by a Zeiss microscope (Axioplan 2), and the viability of the fibroblasts evaluated on the basis of the cell shape and staining pattern.

\section{Assessment of Wound Closure}

Photographs of the hematoxylin-eosin (H/E) stained wounded cultures were taken by a Zeiss microscope

Table 1 Primary antibodies used for immunohistochemical staining of tissue sections

\begin{tabular}{|c|c|c|}
\hline Sctions & Antibody designation & Source $^{\mathrm{a}}$ \\
\hline \multirow[t]{10}{*}{ Frozen } & Laminin 5 (P3E4) & $\begin{array}{l}\text { Chemicon, Temecula, CA, } \\
\text { USA }\end{array}$ \\
\hline & Collagen IV (PHM12) & $\begin{array}{l}\text { Chemicon, Temecula, CA, } \\
\text { USA }\end{array}$ \\
\hline & Collagen VII (LH7.2) & $\begin{array}{l}\text { Dr IM Leigh, London, } \\
\text { England }\end{array}$ \\
\hline & BP180EC (NCC-Lu-226) & $\begin{array}{l}\text { Dr S Hirohashi, Tokyo, } \\
\text { Japan }\end{array}$ \\
\hline & 120/97-kD LAD-1 (123) & $\begin{array}{l}\text { Dr MP Marinkovich, CA, } \\
\text { USA }\end{array}$ \\
\hline & Uncein (19-DEJ-1) & $\begin{array}{l}\text { Dr JD Fine, Chapel Hill, } \\
\text { USA }\end{array}$ \\
\hline & $\alpha_{6}$ chain (JEB5) & $\begin{array}{l}\text { Dr A Sonnenberg, } \\
\text { Amsterdam, The } \\
\text { Netherlands }\end{array}$ \\
\hline & $\beta_{4}$ chain (3E1) & Biomol, Hamburg, \\
\hline & & Germany \\
\hline & RS/DJ-1 (E2.19) & Dr Y Hod, New York, USA \\
\hline \multirow[t]{4}{*}{$\begin{array}{l}\text { Paraffin- } \\
\text { embedded }\end{array}$} & Keratin 10 (DE-K10) & $\begin{array}{l}\text { ICN Biomedicals Inc, } \\
\text { Aurora,OH, USA }\end{array}$ \\
\hline & Keratin 16 (LL0025) & $\begin{array}{l}\text { Dr IM Leigh, London, } \\
\text { England }\end{array}$ \\
\hline & Keratin 17 (CK-E3) & Sigma, S Louis, MO, USA \\
\hline & SKALP & $\begin{array}{l}\text { Dr J Schalkwijk, } \\
\text { Nijmegen, The } \\
\text { Netherlands }\end{array}$ \\
\hline
\end{tabular}

a Antibodies not purchased from indicated sources were personal gifts from the investigator named. and re-epithelialization assessed by measuring the length of the wound covered by the newly formed epidermis using the AxioVision 3.0 program (Zeiss, Hallbergmoos, Germany). The wounding in the central region of the culture enabled the measurement of re-epithelialization from both wound margins.

\section{Cellular Protein Extraction}

For the re-epithelialization experiments, large reconstructed skin cultures were used. After a 2-week culture at the $\mathrm{A} / \mathrm{L}$ interface the cultures were separated into three different regions: (1) central, (2) newly epithelialized region close to the central and (3) re-epithelialized region remote from the central region. The epidermal layer was then mechanically separated from the dermal compartment by forceps and homogenized using a Polytron (PT3000, Kinematica AG). Epidermal proteins were subsequently isolated using TRIzol $^{\circledR}$, (GibcoBRL/ LifeTechnologies) following the manufacturer's instructions. The air-dried protein pellets were solubilized in $9 \mathrm{moll}^{-1}$ urea, $2 \%$ CHAPS, $0.5 \%$ IPG buffer nonlinear gradient of $\mathrm{pH}$ 3-10 (Amersham Pharmacia Biotech, Roosendaal, The Netherlands) and $5 \mathrm{mg} \mathrm{ml}^{-1}$ dithiothreitol. Protein concentrations were determined using the modified Bradford protein assay. ${ }^{28}$

\section{Two-dimensional Polyacrylamide Gel Electrophoresis}

A trace of bromophenol blue was added to the protein samples and known amounts of protein were loaded on 18-cm isoelectric focusing readymade IPG strips with a nonlinear gradient of $\mathrm{pH} 3-$ 10 (Amersham Pharmacia Biotech). Rehydration of the IPG strips was performed for $12 \mathrm{~h}$ at $30 \mathrm{~V}$ after which proteins were focused for $65000 \mathrm{Vh}$ (IPGphor, Amersham Pharmacia Biotech). Prior to the second dimension, IPG strips were equilibrated in $1 \%$ dithiothreitol (w/v) followed by $2.5 \%$ iodoacetamide $(\mathrm{w} / \mathrm{v})$, both for $15 \mathrm{~min}$ in $50 \mathrm{mmol}^{-1}$ Tris-HCl $\mathrm{pH} 8.8,6 \mathrm{moll}^{-1}$ urea, $30 \%$ glycerol and $2 \%$ SDS. After this procedure, the strips were placed on top of a $200 \times 260 \times 1 \mathrm{~mm}^{3}$ polyacrylamide gel $(13 \%$ homogeneous, $2.6 \%$ cross-linking, $0.1 \%$ SDS, $375 \mathrm{mmol}^{-1}$ Tris-HCl pH 8.8) and run for $45 \mathrm{~min}$ at $5 \mathrm{~W} / g e l$ followed by $4 \mathrm{~h}$ at $15 \mathrm{~W} / g e l$ (Ettan Dalt, Amersham Pharmacia Biotech). Proteins were visualized using silver staining ${ }^{29}$ and analysed by PD-Quest software (BIO-RAD Laboratories, Veenendaal, The Netherlands).

\section{Peptide Mapping and Sequencing}

Protein spots of interest were excised from the gels, cut into pieces and dehydrated in $100 \%$ acetonitrile for $10 \mathrm{~min}$. After removal of the acetonitrile, gel 
pieces were dried in a Speed-Vac (Savant Instruments, Holbrook, NY, USA) and subsequently allowed to reswell in a trypsin solution $\left(20 \mathrm{ng} \mu \mathrm{l}^{-1}\right.$ (Promega Benelux, Leiden, The Netherlands) in $50 \mathrm{mmol} \mathrm{l}^{-1}$ ammonium bicarbonate) for $45 \mathrm{~min}$ on ice. Further incubation was performed overnight at $37^{\circ} \mathrm{C}$. Peptide fragments were extracted for $20 \mathrm{~min}$ using $100 \mu \mathrm{l} 25 \mathrm{mmoll}^{-1}$ ammonium bicarbonate, followed by three extractions using $100 \mu \mathrm{l} 5 \%$ formic acid $/ 50 \%$ acetonitrile. The extraction solutions were pooled, concentrated to approximately $30 \mu \mathrm{l}$ (SpeedVac, Savant Instruments) and desalted over Poros 50R2 (Applied Biosystems, Foster City, CA, USA). Peptides were eluted in $60 \%$ methanol $/ 5 \%$ formic acid and measured directly on a Q-TOF electrospray ionization time-of-flight mass spectrometer (Micromass, Manchester, UK).

\section{Results}

\section{Fibroblasts Facilitate Wound Closure}

In order to study wound closure in vitro, HSE were injured using two approaches: (a) 'full-thickness' wounding introduced by freezing with liquid nitrogen or (b) superficial incisional wounding by which only the epidermis has been removed. The first treatment resulted in a blister formation covering the wound. In the injured epidermis, the cell death occurred as judged from the presence of small round-shaped cells (Figure 1a). In the dermal compartment, the majority of the fibroblasts did not survive the low-temperature treatment, as established by trypan blue staining and rounded cell shape (Figure 1b). In contrast, superficial incisional wounding did not lead to the depletion of the fibroblasts from the dermal compartment in the injured part of the culture and the fibroblasts showed an elongated shape and did not positively stain with trypan blue (Figure 1c, d). Repopulation of the dermal compartment with fibroblasts in fullthickness wounds occurred earlier in the presence of EGF than in the absence of growth factors or presence of KGF (data not shown). During subsequent cultures at the $\mathrm{A} / \mathrm{L}$ interface, re-epithelialization from the wound edges occurred in both models (Figure 1a, c) but was faster in superficial than fullthickness wounds (Figure 2a). The addition of growth factors during the re-epithelialization process accelerated the wound closure of freezeinduced wounds, with EGF being slightly more effective than KGF (Figure 2b). The effect of EGF and KGF was most pronounced on day 7 after injury and to a lesser extent at day 14, probably due to the effect of fibroblasts starting to migrate from the wound margins into the wounded regions (data not shown). The effect of growth factors was marginal in superficial incisional wounds (Figure 2c). At day 7, the extent of wound closure in superficial wounds was similar to that found in EGF- or KGF-treated fullthickness wounds (Figure 2b, c). In the uninjured

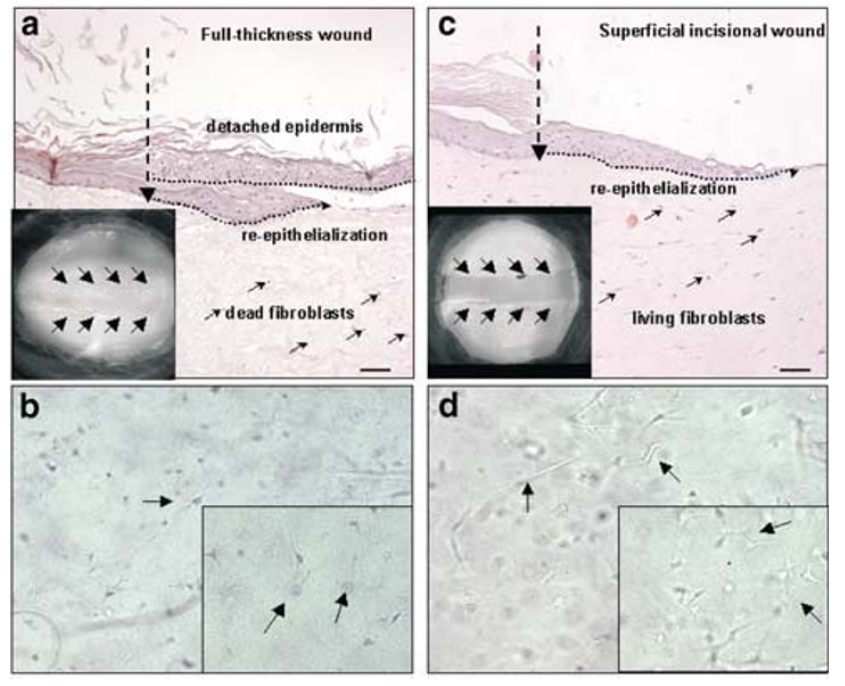

Figure 1 Wounding of human skin equivalents generated on fibroblast-populated collagen matrices. HSE were established by culturing human keratinocytes on fibroblast-populated collagen matrices for 2 weeks at the air-liquid interface in serum-free medium and in the absence of growth factors. Thereafter, the cultures were wounded. (a) 'Full-thickness' wound introduced by freezing with liquid nitrogen or (c) superficial incisional wound by which only the epidermis was removed. After wounding, the cultures were further grown at the air-liquid interface in a serumfree medium. Re-epithelialization from the wound edges 3 days after wounding is shown. Note: (a) In full-thickness wounds cell death occurred in the injured epidermis, as judged from the presence of small round-shaped cells. The dashed perperdicular arrow denotes the wound edge. Inlet: macroscopic view of the injured HSE (arrows indicate the wound margins). (b) In the dermal compartment, the majority of the fibroblasts did not survive the low-temperature treatment, as established by trypan blue staining and the appearance of rounded cells in the dermal compartment (arrows:). (c) Incisional wounding of the epidermis did not affect fibroblast viability. The dashed perperdicular arrow denotes the wound edge. Inlet: macroscopic view of the injured HSE (arrows indicate the wound margins). (d). Notice the differences in the epidermal architecture between different cultures. Hematoxylin- and eosin-stained paraffin cross-sections are shown. Scale bar $(\mathbf{a}, \mathbf{b}): 50 \mu \mathrm{m}$. Specimens were incubated for 2 min in $5 \%$ trypan blue in PBS and thereafter washed with PBS $(\mathbf{c , d}$ : magnifications $\times 100$ and $\times 200)$.

epidermis, keratin 10 was expressed in all suprabasal layers, involucrin in the stratum granulosum and keratins 16 and 17, and skin-derived antileukoproteinase (SKALP) were absent (data not shown). In reepithelialized regions, suprabasal keratin 10 expression persisted, but abundant suprabasal expression of keratins 16 and 17, SKALP and involucrin was noticed (data not shown). This occurred irrespective of the absence or presence of growth factors (data not shown), similarly as seen previously at the periphery of uninjured culture.

\section{Preservation of Laminin 5 Depends on the Wounding Procedure}

Prior to injury, laminin 5 was deposited along the entire DEJ. As shown in Figure 3a, in full-thickness 
106
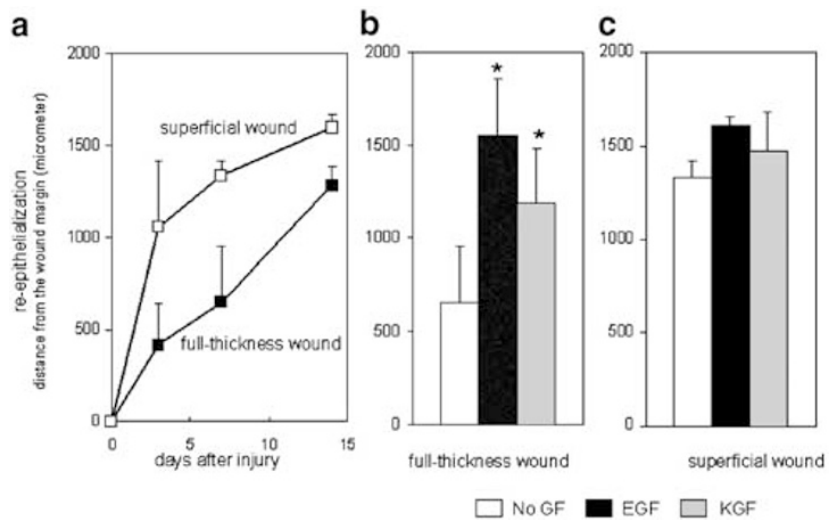

Figure 2 Re-epithelialization of wounded cultures. HSE were established by culturing human keratinocytes on fibroblastpopulated collagen matrices for 2 weeks at the air-liquid interface in a serum-free medium and in the absence of growth factors. Thereafter, the cultures were wounded. (a) Re-epithelialization in full-thickness and superficial incisional wounds was assessed by measuring the length of the wounds covered by the newly formed epidermis on days 3, 7 and 14 after injury. The wound closure in the absence of growth factors is shown. Note: the re-epithelialization process proceeded more rapidly in superficial wounds. After wounding, the HSE were grown in the absence or presence of growth factors ( $1 \mathrm{ng} \mathrm{ml}^{-1} \mathrm{EGF}$ or $5 \mathrm{ng} \mathrm{ml}^{-1} \mathrm{KGF}$ ). (b) The addition of growth factors during the re-epithelialization process accelerated the wound closure of full-thickness wounds, the EGF being more effective than KGF. (c) Growth factors had a marginal effect in superficial incisional wounds. Data 7 days after injury are shown. The values indicate the mean \pm s.e.m. of two independent experiments, each performed in duplicate. ${ }^{*} P<0.05$.

wounds introduced by liquid nitrogen, laminin 5 decorated entirely the DEJ of the newly formed epidermis already in early stages after wounding. Furthermore, laminin 5 was also decorating the surface of the dermal compartment in the wounded area, indicating that after wounding some of the BM components remain associated with the epidermis and some with the dermal part (Figure 3a). In a few cases, laminin 5 also decorated the plasma membrane of the basal keratinocytes in detached epidermis (Figure $3 \mathrm{~b}$ ).

In contrast, in superficial wounds created by mechanical removal of the epidermis, laminin 5 was absent at early time points (3-4 days after injury; notice the absence of the stratum corneum) during re-epithelialization (Figure 3c). At later time points (at days 7 and 14; notice the presence of the stratum corneum), laminin 5 decorated the DEJ (Figure 3d inlet). Evaluation at day 14 revealed a weak expression of collagen type VII (Figure 3d) and abundant expression of $\alpha_{6}$ and $\beta_{4}$ integrin subunits (Figure 3e, f).

\section{Identification of Proteins during Epithelialization}

A different approach was chosen to study the changes in epidermal protein profiles during epithelialization. HSE were generated by seeding kerati-

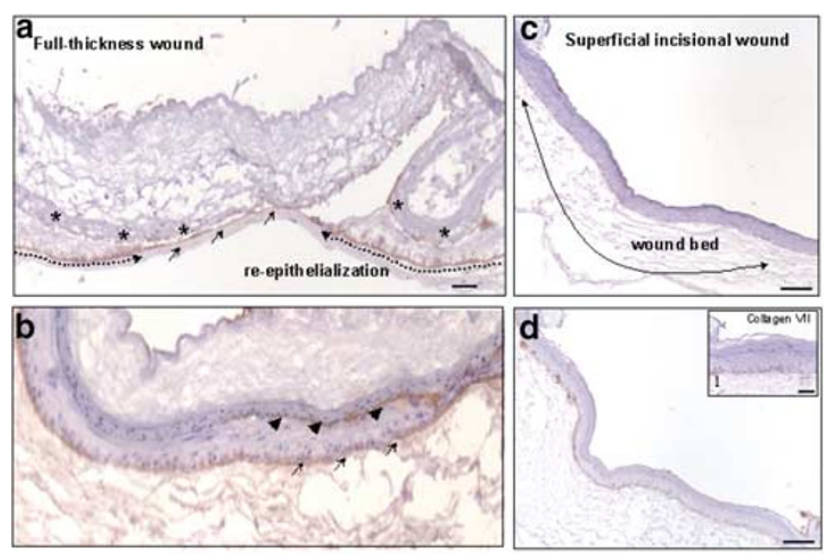

Figure 3 Expression of various basement membrane proteins during re-epithelialization of full-thickness $(\mathbf{a}, \mathbf{b})$ and superficial wounds (c,d). HSE were established by culturing human keratinocytes on fibroblast-populated collagen matrices for 2 weeks at the air-liquid interface in a serum-free medium and in the absence of growth factors. Thereafter, the cultures were wounded. immunohistochemical staining using antibodies directed against human laminin 5, collagen type VII is shown. (a) In full-thickness wounds introduced by liquid nitrogen laminin 5 decorated the entire DEJ of the newly formed epidermis already in early stages after wounding (3-4 days after injury) (dashed arrows) and the surface of the dermal compartment in the wounded area (arrows). In the injured epidermis cell death occurred as judged from the presence of small round-shaped cells (asterisks). (b) Laminin 5 decorated DEJ of the newly formed epidermis (arrows) and also the plasma membrane of the basal keratinocytes in detached epidermis (arrowheads). (c,d) In superficial wounds laminin 5 was absent at early time points (C, 3 to 4 days after injury) at which re-epithelialization was observed, but it was decorating the DEJ at day 7 (d). At day 14 weak expression of collagen type VII (d, inlet) was observed. Scale bar: a, b, D1,: $50 \mu \mathrm{m} ; \mathbf{c}, \mathbf{d}: 100 \mu \mathrm{m}$.

nocytes onto the central area (diameter $2 \mathrm{~cm}$ ) of a large fibroblast-populated collagen matrix (diameter $7.5 \mathrm{~cm}$ ). During the subsequent 2-week culture at the A/L interface, the keratinocytes differentiate, proliferate and migrate from the initial seeding area to the culture periphery (Figure 4). The epithelialization process is similar to that occurring during reepithelialization after wounding. Therefore, this model can be used to clarify proteins that are involved in re-epithelialization. The cultures were separated into two major regions based on histological criteria: (A) central region, in which the keratinocytes were seeded. In this region, the epidermis was fully differentiated consisting of 8-10 viable cell layers and coherent stratum corneum. (B) the laterally expanding regions (B1-B4) (Figure 4), in which the number of viable cell layers and the thickness of the stratum corneum was gradually decreasing. Stratum corneum was absent in region B3, and in the outermost region B4 only one cell layer was present. Differences in terminal epidermal differentiation (Table 2) and BM formation (Table 3) were noticed between the central and expanding regions: in the central region, keratin 10 was expressed in all suprabasal cell layers and 

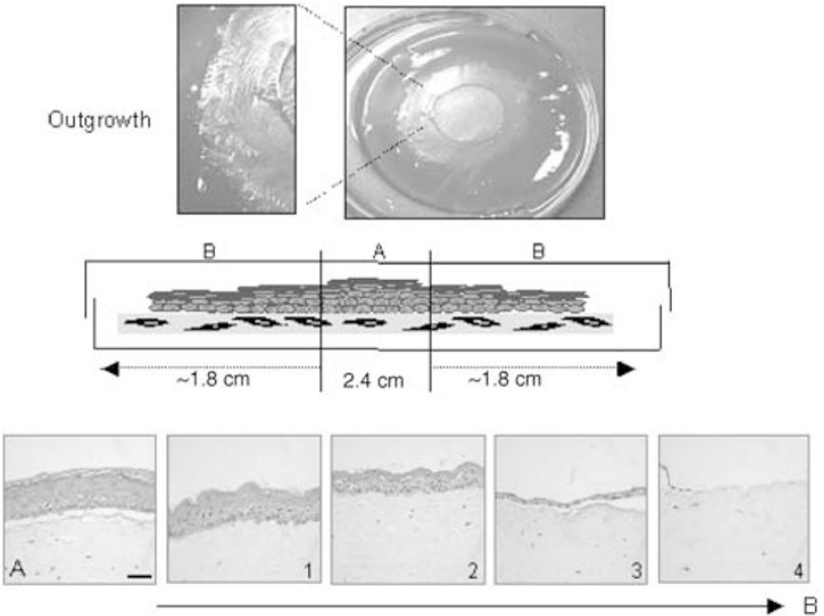

Figure 4 Epithelialization in HSE grown on fibroblast-populated collagen matrix. From the initial seeding area to the culture periphery keratinocytes migrated, proliferated and differentiated. Macroscopical and histological images of reconstructed epidermis established on collagen matrices populated with $1 \times 10^{5}$ fibroblasts $\mathrm{ml}^{-1}$ (diameter, $7.5 \mathrm{~cm}^{2}$ ) after 2-week culture at the air-liquid interface in serum-free medium and in the absence of growth factors. The cultures were separated into two major regions based on histological criteria: (a) central region, (b) the laterally expanding regions (1-4), as schematically depicted in the middle figure Hematoxylin- and-eosin stained paraffin crosssections are shown in the lower figure. Note: the number of viable cell layers and the thickness of the stratum corneum in the different regions. Scale bar: $50 \mu \mathrm{m}$.

keratins 6 and 16 were expressed in the basal cell layer and their expression shifted towards the stratum granulosum. K17 was absent in all epidermal cell layers. The terminal differentiation marker involucrin was expressed in the stratum granulosum, whereas SKALP was absent (data not shown). In laterally expanding regions, suprabasal keratin 10 expression persisted, but abundant suprabasal expression of keratins 16 and 17 was noticed. Similar observations were made for SKALP and involucrin (data not shown). While no difference in the deposition of BP180 EC, BP230 and plectin was observed in laterally expanding epidermis as compared to the central region, LAD-1 expression was reduced and that of uncein was very faint and patchy in expanding epidermal tissue (Table 3 and Figure 5). In the outermost expanding regions no laminin 5 deposition was observed (Figure 5). There also keratin 10, involucrin and SKALP could not be detected (data not shown). The deposition of type IV and VII collagen was hardly detectable in the laterally expanding regions (Table 3), while integrin subunits $\alpha_{6}, \beta_{4}$ and BP180 EC were abundantly expressed (Figure 5).

As with immunohistochemistry only the expressional changes of known proteins during re-epithelialization can be monitored, the 2D-gel electrophoresis approach has been chosen to obtain more detailed information on changes in the overall
Table 2 Expression of specific differentiation protein markers in human skin equivalents during epithelialization process

\begin{tabular}{|c|c|c|c|c|}
\hline Scaffold & Native skin & $\operatorname{HSE}(a)$ & $H S E(b)$ & $H S E(c)$ \\
\hline \multicolumn{5}{|l|}{ K10 } \\
\hline \multicolumn{5}{|l|}{ SG } \\
\hline \multicolumn{5}{|l|}{ SS } \\
\hline \multicolumn{5}{|l|}{ SB } \\
\hline \multicolumn{5}{|l|}{ K6 } \\
\hline \multicolumn{5}{|l|}{ SG } \\
\hline \multicolumn{5}{|l|}{ SS } \\
\hline \multicolumn{5}{|l|}{ SB } \\
\hline \multicolumn{5}{|l|}{ K16 } \\
\hline \multicolumn{5}{|l|}{ SG } \\
\hline \multicolumn{5}{|l|}{ SS } \\
\hline \multicolumn{5}{|l|}{ SB } \\
\hline \multicolumn{5}{|l|}{ K17 } \\
\hline SG & & & & \\
\hline SS & & & & \\
\hline SB & & & & \\
\hline
\end{tabular}

\begin{tabular}{|l|l|l|l|l|l|l}
\hline & & & & & & \\
\hline
\end{tabular} Human skin equivalents (HSE) were generated by seeding of keratinocytes onto the central region of fibroblast-populated collagen matrices and subsequent culturing for 2 weeks at the A/L interface in the absence of growth factors. Thereafter, the cultures were separated into three different regions (a) the central fully differentiated region, (b) newly epithelialized region close to the central and (c) reepithelialized region remote from the central region. The expression of various proteins was assessed by immunohistochemistry. Two independent observers performed the evaluation of staining localization and intensity.

K, keratin; SB, stratum basale; SS, stratum spinosum; SG, stratum granulosum.

protein spectrum. For this purpose, proteins were isolated from different regions of the laterally expanding epidermis and subjected to 2D-PAGE, with the exception of the outermost region from which sufficient amounts of required material could not be harvested. The epidermal protein samples were run on parallel 2D-gels and visualized using silver staining. 2D-PAGE protein profiles obtained showed great similarities, indicating high reproducibility of the system. A representative 2D gel image is shown in Figure 6a. After scanning, gel images were analyzed by PD-Quest (BIO-RAD Laboratories) for spots that increased or decreased in different stages of the migration. Different spots were found to vary in intensity during the epithelialization process. As we observed interexperimental/donor variations in the intensity of various spots, only spots that showed similar changes in intensity in four independent experiments, performed with HSE generated with cells originating from four different skin donors, were selected for further analysis. In total, three spots were identified, of which the density was found to increase or decrease in different stages of the migration. The protein that fitted best to the criteria of selection was annotated as spot SSP4209 (Figure 6a). The intensity of the 
Table 3 Expression of basement membrane and hemidesmosomal proteins in human skin equivalents during the epithelialization process

\begin{tabular}{llll}
\hline & Native skin & HSE(a) \\
Collagen VII & & \\
SB & & \\
DEJ \\
Derm
\end{tabular}

From the negative to a strong positive expression T Patchy expression Human skin equivalents (HSE) were generated by seeding of keratinocytes onto the central region of fibroblast-populated collagen matrices and subsequent culturing for 2 weeks at the A/L interface in the absence of growth factors. Thereafter, the cultures were separated into three different regions (a) the central fully differentiated region, (b) newly epithelialized region close to the central and (c) reepithelialized region remote from the central region. The expression of various basement membrane and hemidesmosomal proteins was assessed by immunohistochemistry. Two independent observers have performed the evaluation of staining localization and intensity. SB,stratum basale; DEJ, dermal-epidermal junction, derm, dermal substrate. spot decreased from the central region towards the periphery of the cultures (Figure 6b). SSP4209 was also present in native skin. After tryptic digestion of SSP4209, mass spectrometry was used to characterize the protein (Figure 6c). Several unique fragments were detected and sequence analysis of one of these fragments unambiguously showed that spot SSP4209 corresponds to RS/DJ-1 (MW 19.89 kDa, pI about 6.33). Mass spectrometry of the SSP4209 spot was performed using material derived from two different donors in two independent experiments. Both sequence analyses identified SSP4209 as RS/ DJ-1.

\section{Immunohistochemical Confirmation of the RS/DJ-1 Protein during Re-epithelialization}

To examine the localization and potential redistribution of the RS/DJ-1 protein during the epithelialization process, immunohistochemistry was performed on the same skin specimens that had been used to isolate proteins for 2D-PAGE analysis. In native skin and the central HSE region, a strong cytoplasmic and nuclear RS/DJ-1 staining in the entire epidermis was observed (Figure 7). In the expanding regions, only the stratum granulosum stained negatively, while in the outermost outgrowing region the presence of DJ-1 could not be detected. These results confirmed the results obtained with 2D-PAGE analysis.

\section{Discussion}

Re-epithelialization of a wound primarily involves the migration of keratinocytes from the edges of the wound and hair follicles, their proliferation, differentiation and stratification to form the neo-epithelium. ${ }^{30}$ During this process, keratinocyte migration and proliferation will depend on their interaction with dermal fibroblasts and with the extracellular matrix $^{31-33}$ and on a variety of growth factors and cytokines present in wound fluid. ${ }^{34}$ In this study, we have established an in vitro human wound-healing model, which enables us to examine the role of fibroblasts and exogenous growth factors on wound re-epithelialization. In this reconstructed human skin model generated by culturing keratinocytes on fibroblast-populated collagen matrix, epidermal stratification is reproduced to a high extent, similar to that occurring in vivo. ${ }^{20,23,35}$ Our recent study ${ }^{26}$ showed that when HSE are grown in serum-free media and in the absence of growth factors, the number and the functional state of fibroblasts incorporated into the collagen matrix is crucial for the normalization of the epidermal differentiation program and of the deposition of various basement membrane and hemidesmosomal components. To examine the potential role of fibroblasts in the reepithelialization process, two types of wounds were introduced in our HSE: superficial wounds, in 

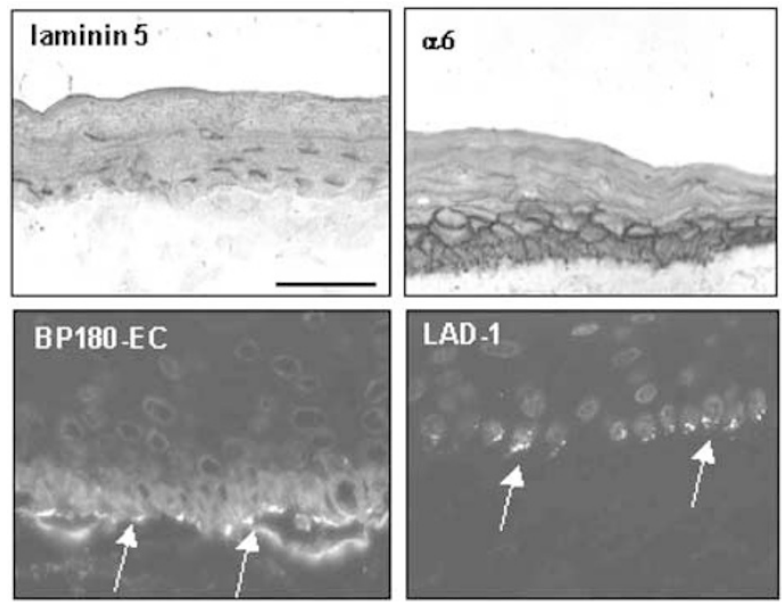
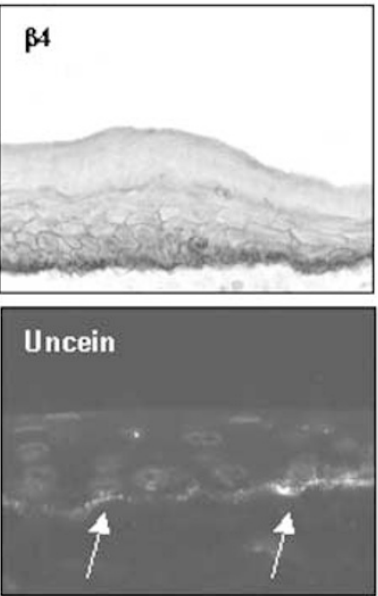

Figure 5 Differential expression of basement membrane and hemodesmomal proteins in laterally expanding keratinocyte regions on fibroblast populated collagen matrices. HSE were generated by seeding keratinocytes onto the central area (diameter 2 cm) of a large fibroblast-populated collagen matrix (diameter $7.5 \mathrm{~cm}$ ). During the subsequent 2-week culture at the A/L interface, the keratinocytes migrate from the initial seeding area to the culture periphery and proliferate and differentiate. Immunofluorescence and immunohistochemical staining of antibodies directed against laminin 5, integrin subunits $\alpha_{6}$ and $\beta_{4}$, BP180-EC, $127-\mathrm{kD}$ LAD-1 and uncein (DEJ-19) are shown. In the latterly expanding epidermis no laminin 5 expression was observed, while the integrin-subunit $\alpha_{6}$ was abundantly expressed in several suprabasal cell layers. Integrin-subunit $\beta_{4}$ and BP180-EC were expressed only in the first suprabasal cell layer. In expanding epidermal tissue, LAD-1 expression was weak and that of uncein was very faint and patchy. Scale bar: $50 \mu \mathrm{m}$.

which the underlying dermal compartment remained populated with functional fibroblasts, and full-thickness wounds, in which fully functional fibroblasts were present only in uninjured regions. As in vivo, also in vitro re-epithelialization of superficial wounds proceeded faster than that of full-thickness wounds, indicating the accelerating effect of fibroblasts in this process. Fibroblastderived soluble factors released from fibroblasts present in uninjured regions in the full-thickness wound model were, however, not sufficient to support keratinocyte migration and proliferation to such an extent as fibroblasts present in the dermal regions underlying the injured epidermis in superficial wounds. These findings indicate that the epithelial-mesenchymal interaction plays an important role in establishing the profile of released factors regulating proliferation and differentiation of keratinocytes. This interaction can be based on (i) production of soluble factors by either epithelial or mesenchymal cells that exhibit autocrine and/or paracrine activities, ${ }^{24,27,36-40}$ (ii) cell-matrix interactions $^{41}$ (iii) signaling by direct cell-cell contact. ${ }^{42}$ The lower re-epithelialization rate in full-thickness wounds is indicative that in addition to paracrine cytokine and growth factor action, the crosstalk between keratinocytes and fibroblasts are important regulators of keratinocyte proliferation and differentiation. Administration of exogenous EGF or KGF facilitated wound closure only in the full-thickness wounds, in which the injured dermal compartment was deprived of viable fibroblasts. We noticed, however, that after 3 days, re-epithelialization was lower in full-thickness wounds cultured with KGF or EGF compared to superficial wounds (data not shown). This finding indicates that other growth factor(s) or cytokine(s) released during keratinocytefibroblast crosstalk contribute to the wound closure. These factors may include TGF- $\alpha$ (5), TGF- $\beta,{ }^{43}$ GM$\mathrm{CSF}^{44}{ }^{4}$ bGFG,${ }^{45} \mathrm{HGF}^{46}$ IL-6 and IL- $1 .{ }^{36}$

In contrast to the enhanced re-epithelialization of superficial incisional wounds, the deposition of DEJ was delayed (day 7 postinjury). In full-thickness wounds laminin 5 decorated the apical surface of the dermal compartment. On a few occasions, laminin 5 also decorated the lower site of the basal keratinocytes in the detached epidermis. This finding indicates that during freeze injury, some BM components remain associated with the dermal compartment and probably facilitate the BM reconstitution. Similar preservation of the BM components has been noticed upon separation of the epidermis and dermis from native skin by incubation in calcium, magnesium-free buffer at $37^{\circ} \mathrm{C}$, the method used for the preparation of the de-epidermized dermis. ${ }^{47,48}$

As an alternative approach to study the expression of basement membrane proteins during reepithelialization, we analyzed their expression in different laterally expanding regions on an unwounded HSE. During the epithelialization process no laminin 5, type IV and VII collagen deposition was detected in the outermost expanding regions. In contrast to this, the $\alpha_{6} \beta_{4}$ integrin that serves as a receptor for laminin 5 was expressed in the outermost regions of the expanding epidermis. In these regions, the expression of integrin subunit $\beta_{1}$ was downregulated (data not shown). These findings differ from the in vivo situation where it has been shown that $\alpha_{3} \beta_{1}$ integrin is ubiquitously deposited 

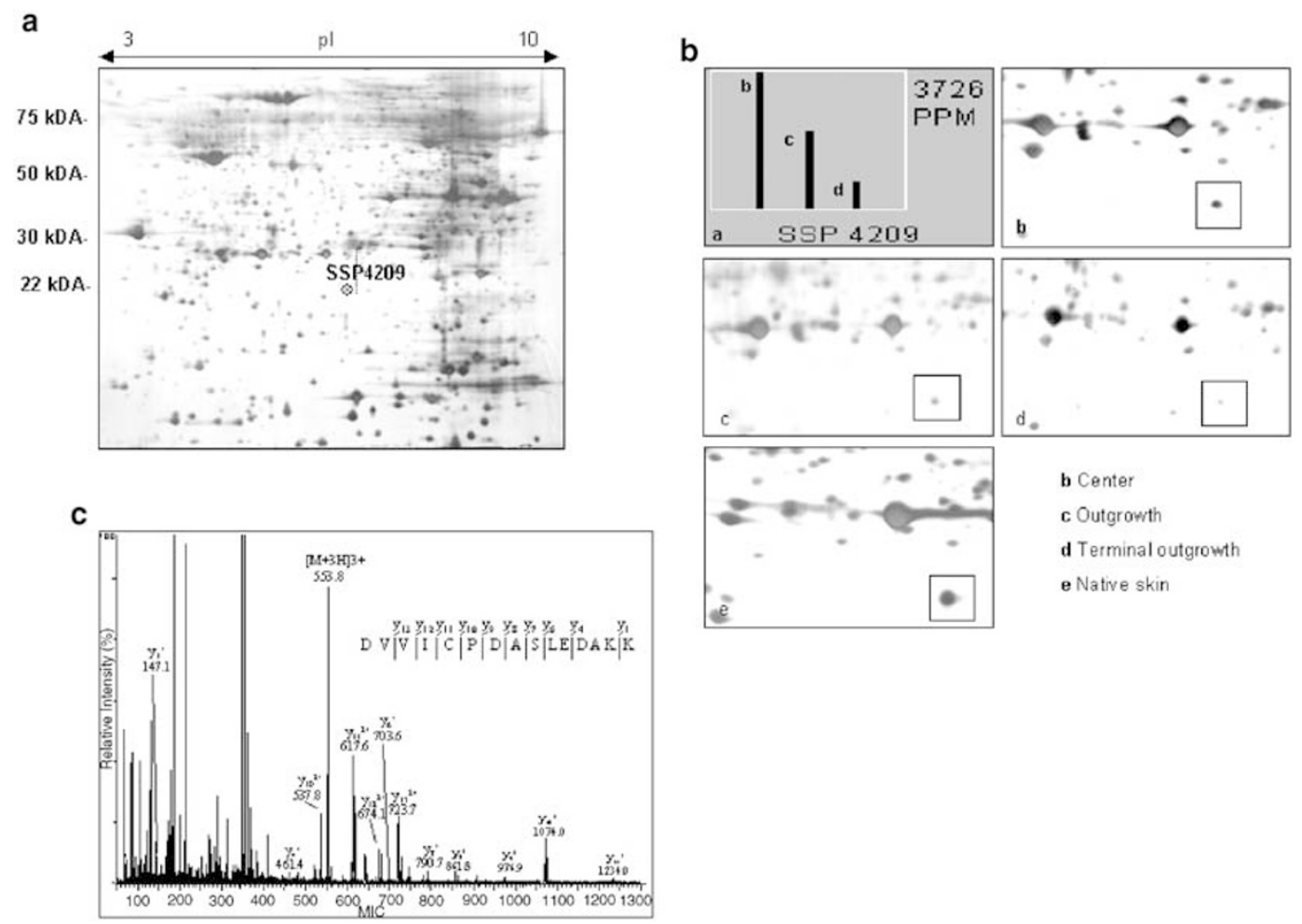

Figure 6 Identification of differential expressed proteins during the re-epithelialization process using two-dimensional polyacrylamide gel electrophoresis (2D-PAGE). HSE were generated by seeding keratinocytes onto the central area (diameter $2 \mathrm{~cm}$ ) of a large fibroblastpopulated collagen matrix (diameter $7.5 \mathrm{~cm}$ ). During the subsequent 2-week culture at the A/L interface the keratinocytes migrate from the initial seeding area to the culture periphery, proliferate and differentiate. Proteins were isolated from three different regions of the laterally expanding epidermis (b: central, c: outgrowth; d: terminal outgrowth) and from the native epidermis (e) and separated by $2 \mathrm{D}-$ PAGE. (a) A representative 2D-PAGE image representing epidermal proteins expressed in the central area of the HSE. Protein of interest is designated as spot SSP4209 (indicated with a circle). (b) (a) The relative density of spot SSP4209 in the regions b-d, as determined by the PD-Quest software. Higher magnification of boxed area (shown in A) showing the decreased expression of spot SSP4209 from the central region (b) towards the expanding regions (c-d); (e) expression in native epidermis. (c) Identification of spot SSP4209 as DJ-1. The MS/MS spectrum of ion species at $\mathrm{m} / \mathrm{z} 553.8(\mathrm{M}+3 \mathrm{H}) 3+$ generated after tryptic digestion of spot SSP4209 is shown. The major ion series indicated in the spectrum are attributable to single and doubly charged $y$-ions of the peptide DVVICPDASLEDAKK, corresponding to a tryptic fragment of DJ-1.

by keratinocytes during migration and that it inhibits keratinocyte migration. ${ }^{49}$ Furthermore, the $\alpha_{3} \beta_{1}$ integrin is also thought to function as a receptor for laminin $5 .^{50}$ Overall, our findings indicate that the presence of $\beta_{1}$ integrin, laminin 5, type IV and VII collagen in the laterally expanding epidermis is not required for keratinocyte migration in vitro. This is in contrast to the results obtained by Laplante et al, ${ }^{18}$ who wounded the HSE with $6 \mathrm{~mm}$ biopsy punch and subsequently placed it over a fibroblast sheet. They observed the deposition of laminin 5 and collagen IV at the DEJ already 3 days after wounding. However, this may be attributed to the presence of exogenous EGF ( $10 \mathrm{ng} \mathrm{ml}^{-1}$ ) as we have recently observed that in organotypic keratinocyte monocultures laminin 5 deposition is induced by exogenous EGF, not only at the DEJ but also in the keratinocyte cytoplasm, in the basal and first suprabasal cell layer (El Ghalbzouri et al, submitted).

In contrast to the wounding model, the unwounded HSE also allowed us to collect sufficient material for studying protein expression during epithelialization using 2D-PAGE. For this purpose, tissue from different regions of the expanding epidermis reconstructed on large collagen matrices was collected. Using this approach, we have found three proteins, the amounts of which change during the epithelialization process in four independent experiments performed with cells derived from four different skin donors. From these proteins, DJ-1 was identified as the most prominent protein as its amount was significantly decreasing from the central region towards the periphery of the culture. This observation was confirmed by immunohistochemistry using monoclonal antibody directed against RS (DJ-1). In native skin and in the central HSE (seeding) region, a strong cytoplasmic and nuclear expression of RS/DJ-1 was observed in the entire epidermis. In the laterally expanding regions, the staining intensity and localization of DJ-1 were gradually changing. In the regions close to the central one, positive DJ-1 staining was detected in all cell layers, except the stratum granulosum. In the most remote regions DJ-1 was absent.

To our knowledge we are the first group reporting a differential expression of DJ-1 in the epithelializa- 

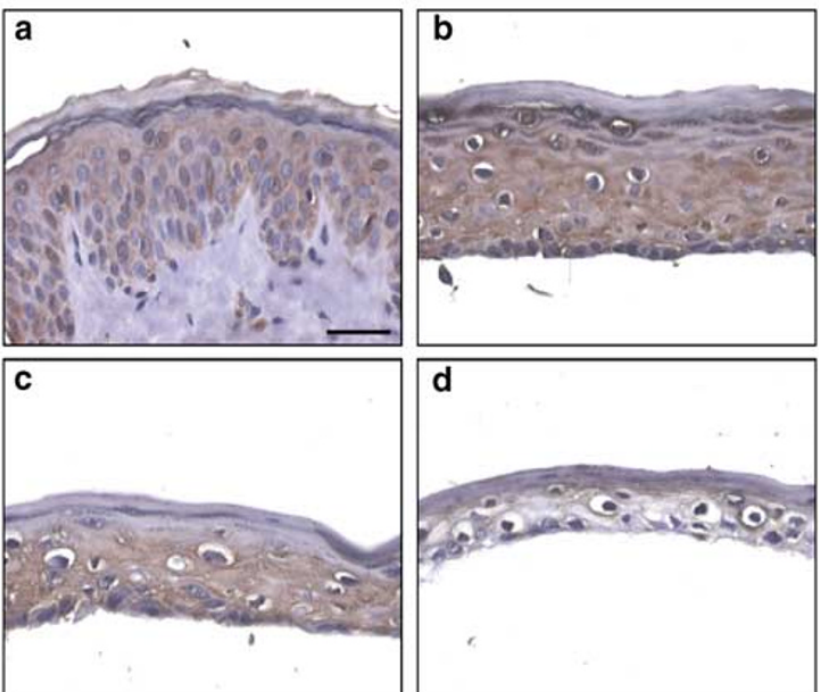

Figure 7 RS/DJ-1 protein expression decreases during re-epithelialization. Immunohistochemical stainings of DJ-1 during the epithelialization process are shown. (a) Native skin; (b) center of the HSE; (c) outgrowth; (d) terminal outgrowth. Note that the strong cytoplasmic and nuclear RS/DJ-1 staining in the entire epidermis was observed in native and central areas of HSE. Scale bar: $50 \mu \mathrm{m}$.

tion process. However, it is difficult to speculate on its role in this process because the function of DJ-1 in still unknown. DJ-1 was first identified as a protein that transforms mouse NIH3T3 cells in cooperation with activated ras. ${ }^{51}$ It has been previously demonstrated by Hod and his group that RS is identical to the oncogene DJ-1. ${ }^{52,53}$ In addition to the role in malignant transformation, DJ-1 was also found to play a role in the oxidative stress response ${ }^{54}$ the fertilization process ${ }^{55,56}$ cell transformation, ${ }^{57}$ and as the regulatory subunit of a $400 \mathrm{kDa}$ RNA-binding protein complex. ${ }^{52}$ More recently, structural analysis suggests that DJ-1 may possess an active site, and could be a protease. ${ }^{58}$

In conclusion, the data presented here indicate that the human skin equivalent model serves as a useful in vitro tool for detailed studies on processes and factors controlling the re-epithelialization process during wound closure.

\section{Acknowledgement}

We highly appreciate the generous gift of Dr Hod for the antibody directed against DJ-1.

\section{References}

1 Downing DT. In vivo studies of cutaneous lipid biosynthesis. Semin Dermatol 1992;11:162-168.

2 Bickenbach JR, Greer JM, Bundman DS, et al. Loricrin expression is coordinated with other epidermal proteins and the appearance of lipid lamellar granules in development. J Invest Dermatol 1995;104:405-410.
3 Roop D. Defects in the barrier. Science 1995;267: 474-475.

4 Singer AJ, Clark RA. Cutaneous wound healing. N Engl J Med 1999;341:738-746.

5 Clark RA. Biology of dermal wound repair. Dermatol Clin 1993;11:647-666.

6 Grinnell F. Fibroblasts, myofibroblasts, and wound contraction. J Cell Biol 1994;124:401-404.

7 Martin P. Wound healing-aiming for perfect skin regeneration. Science 1997;276:75-81.

8 Coulombe PA. Towards a molecular definition of keratinocyte activation after acute injury to stratified epithelia. Biochem Biophys Res Commun 1997;236:231-238.

9 Stanley JR, Alvarez OM, Bere Jr EW, et al. Detection of basement membrane zone antigens during epidermal wound healing in pigs. J Invest Dermatol 1981;77: 240-243.

10 Rigal C, Pieraggi MT, Vincent C, et al. Healing of full-thickness cutaneous wounds in the pig. I. Immunohistochemical study of epidermo-dermal junction regeneration. J Invest Dermatol 1991;96: 777-785.

11 Timpl R. Macromolecular organization of basement membranes. Curr Opin Cell Biol 1996;8:618-624.

12 Ekblom M, Falk M, Salmivirta K, et al. Laminin isoforms and epithelial development. Ann NY Acad Sci 1998;857:194-211.

13 Di Colandrea T, Wang L, Wille J, et al. Epidermal expression of collagenase delays wound-healing in transgenic mice. J Invest Dermatol 1998;111: 1029-1033.

14 Bechtel MJ, Wysocki NS, Heidtmann A, et al. Plasminogen activator inhibitor type 2 is expressed in keratinocytes during re-epithelialization of epidermal defects. Br J Dermatol 1998;138:22-28.

15 Croft CB, Tarin D. Ultrastructural studies of wound healing in mouse skin. I Epithelial behaviour. J Anat 1970;106:63-77.

16 Tarin D, Croft CB. Ultrastructural studies of wound healing in mouse skin. II Dermo-epidermal interrelationships. J Anat 1970;106:79-91.

17 Manios A, Tzortzaks H, Minogiannis N, et al. A differential model of wound epithelialization for fullthickness skin defects. Ann MBC 1992;9:3-5.

18 Laplante AF, Germain L, Auger FA, et al. Mechanisms of wound reepithelialization: hints from a tissueengineered reconstructed skin to long-standing questions. FASEB J 2001;15:2377-2389.

19 Mazzalupo S, Coulombe PA. A reporter transgene based on a human keratin 6 gene promoter is specifically expressed in the periderm of mouse embryos. Mech Dev 2001;100:65-69.

20 El Ghalbzouri A, Gibbs S, Lamme E, et al. Effect of fibroblasts on epidermal regeneration. Br J Dermatol 2002;147:230-243.

21 Bell E, Ehrlich HP, Buttle DJ, et al. Living tissue formed in vitro and accepted as skin-equivalent tissue of full thickness. Science 1981;211:1052-1054.

22 Boyce ST, Christianson DJ, Hansbrough JF. Structure of a collagen-GAG dermal skin substitute optimized for cultured human epidermal keratinocytes. J Biomed Mater Res 1988;10:939-957.

23 Ponec M, Weerheim A, Kempenaar J, et al. The formation of competent barrier lipids in reconstructed human epidermis requires the presence of vitamin C. J Invest Dermatol 1997;109:348-355. 
24 Stark HJ, Baur M, Breitkreutz D, et al. Organotypic keratinocyte cocultures in defined medium with regular epidermal morphogenesis and differentiation. J Invest Dermatol 1999;112:681-691.

25 Parenteau NL, Nolte CM, Bilbo P, et al. Epidermis generated in vitro: practical considerations and applications. J Cell Biochem 1991;45:245-251.

26 El Ghalbzouri A, Lamme E, Ponec M. Crucial role of fibroblasts in regulating epidermal morphogenesis. Cell Tissue Res 2002;31:189-199.

27 Smola H, Thiekotter G, Fusenig NE. Mutual induction of growth factor gene expression by epidermal-dermal cell interaction. J Cell Biol 1993;122:417-429.

28 Ramagli LS. Quantifying protein in 2-D PAGE solubilization buffers. Methods Mol Biol 1999;112: 99-103.

29 Shevchenko A, Wilm M, Vorm O, et al. Mass spectrometric sequencing of proteins silver-stained polyacrylamide gels. Anal Chem 1996;68:850-858.

30 Woodley DT, Kalebec T, Banes AJ, et al. Adult human keratinocytes migrating over nonviable dermal collagen produce collagenolytic enzymes that degrade type I and type IV collagen. J Invest Dermatol 1986;86:418-423.

31 Garlick JA, Parks WC, Welgus HG, et al. Re-epithelialization of human oral keratinocytes in vitro. J Dent Res 1996;75:912-918.

32 Pilcher BK, Wang M, Qin XJ, et al. Role of matrix metalloproteinases and their inhibition in cutaneous wound healing and allergic contact hypersensitivity. Ann NY Acad Sci 1999;878:12-24.

33 Steffensen B, Hakkinen L, Larjava H. Proteolytic events of wound-healing coordinated interactions among matrix metalloproteinases (MMPs), integrins, and extracellular matrix molecules. Crit Rev Oral Biol Med 2001;12:373-398.

34 Rennekampff HO, Hansbrough JF, Kiessig V, et al. Bioactive interleukin-8 is expressed in wounds and enhances wound healing. J Surg Res 2000;93:41-54.

35 Ponec M, Weerheim A, Lankhorst $\mathrm{P}$, et al. New acylceramide in native and reconstructed epidermis. J Invest Dermatol 2003;120:581-588.

36 Boxman IL, Ruwhof C, Boerman OC, et al. Role of fibroblasts in the regulation of proinflammatory interleukin IL-1, IL-6 and IL-8 levels induced by keratinocyte-derived IL-1. Arch Dermatol Res 1996;288: 391-398.

37 Boxman IL, Lowik CW, Aarden I, et al. Modulation of IL-6 production and IL-1 activity by keratinocytefibroblast interaction. J Invest Dermatol 1993;101: 316-324.

38 Maas-Szabowski N, Fusenig NE. Interleukin-1-induced growth factor expression in postmitotic and resting fibroblasts. J Invest Dermatol 1996;107: 849-855.

39 Maas-Szabowski N, Shimotoyodome A, Fusenig NE. Keratinocyte growth regulation in fibroblast cocultures via a double paracrine mechanism. J Cell Sci 1999;112:1843-1853.

40 Maas-Szabowski N, Stark HJ, Fusenig NE. Keratinocyte growth regulation in defined organotypic cultures through IL-1-induced keratinocyte growth factor expression in resting fibroblasts. J Invest Dermatol 2000;114:1075-1084.
41 Gailit J, Clark RA. Wound repair in the context of extracellular matrix. Curr Opin Cell Biol 19934;6: 717-725.

42 Waelti ER, Inaebnit SP, Rast HP, et al. Co-culture of human keratinocytes on post-mitotic human dermal fibroblast feeder cells: production of large amounts of interleukin 6. J Invest Dermatol 1992;98: 805-808.

43 Compton C, Tong T, Trookman N, et al. TGF-beta 1 gene expression in cultured human keratinocytes does not decrease with biologic age. J Invest Dermatol 103:127-133.

44 Szabowski A, Maas-Szabowski N, Andrecht S, et al. cJun and JunB antagonistically control cytokine-regulated mesenchymal-epidermal interaction in skin. Cell 2000;103:745-755.

45 Schroder JM. Chemotactic cytokines in the epidermis. Exp Dermatol 1992;1:12-19.

46 Hamoen KE, Morgan JR. Transient hyperproliferation of a transgenic human epidermis expressing hepatocyte growth factor. Cell Transplant 2002;11:385-395.

47 Prunieras M, Regnier M, Woodley D. Methods for cultivation of keratinocytes with an air-liquid interface. J Invest Dermatol 1983;81:28s-33s.

48 Ponec M, Weerheim A, Kempenaar J, et al. Lipid composition of cultured human keratinocytes in relation to their differentiation. J Lipid Res 1988;29:949-961.

49 Zhang K, Kramer RH. Laminin 5 deposition promotes keratinocyte motility. Exp Cell Res 1996;227:309-322.

50 Mayer U, Poschl E, Gerecke DR, et al. Low nidogen affinity of laminin-5 can be attributed to two serine residues in EGF-like motif gamma 2III4. FEBS Lett 1995;365:129-132.

51 Nagakubo D, Taira T, Kitaura H, et al. DJ-1, a novel oncogene product which transforms mouse NIH3T3 cells in cooperation with H-ras. Biochem Biophys Res Commun 1997;231:509-513.

52 Hod Y, Pentyala SN, Whyard TC, et al. Identification and characterization of a novel protein that regulates RNA-protein interaction. J Cell Biochem 1999;72: 435-444.

53 Le Naour F, Misek DE, Krause MC, et al. Proteomicsbased identification of RS/DJ-1 as a novel circulating tumor antigen in breast cancer. Clin Cancer Res 2001;7:3328-3335.

54 Mitsumoto A, Nakagawa Y, Takeuchi A, et al. Oxidized forms of peroxiredoxins and DJ-1 on two-dimensional gels increased in response to sublethal levels of paraquat. Free Radic Res 2001;35:301-310.

55 Wagenfeld A, Gromoll J, Cooper TG. Molecular cloning and expression of rat contraception associated protein 1 (CAP1), a protein putatively involved in fertilization. Biochem Biophys Res Commun 1998;251:545-549.

56 Wagenfeld A, Yeung CH, Shivaji S, et al. Expression and cellular localization of contraception-associated protein. J Androl 2000;21:954-963.

57 Takahashi K, Taira T, Niki T, et al. DJ-1 positively regulates the androgen receptor by impairing the binding of PIASx alpha to the receptor. J Biol Chem 2001;276:37556-37563.

58 Tao X, Tong L. Crystal structure of human DJ-1, a protein associated with early-onset Parkinson's disease. J Biol Chem 2003;278:31372-31379. 